\title{
EMPRENDIMIENTO DE ALTO IMPACTO EN LAS CIUDADES METROPOLITANAS DE BILBAO Y BOGOTÁ D.C. HACIA LA TRANSICIÓN URBANA BIOMIMÉTICA.
}

Hernando Bernal Zamudio, Gloria Marlene Diaz Muñoz, Unai Tamayo Orbegozo, Alex Mitxelena Etxeberria, Daniel Becerra Orcajo, Adalberto Antonio Machado Amador

Universidad del País Vasco UPV/EHU, España; Universidad Externado de Colombia, Bogotá, Colombia; INGEM: Ingeniería y Desarrollo, Getxo, España; Consultor Ministerio de Interior, Bogotá, Colombia.zamazonia@gmail.com,gloria.diaz@uexternado.edu.co,unai.tamayo@ehu.eus, alex.mitxelena@ehu.eus, danbece@ingedemo.com, aama65@hotmail.com

Palabras Clave: Ecosistemas, Emprendimiento, Biomimesis, economía circular, bioarquitectura y ecourbanismo.

\footnotetext{
"EI mundo como lo hemos creado es un proceso de nuestro pensamiento. No puede ser cambiado sin cambiar nuestro pensamiento" (Albert Einstein)
}

\section{Resumen}

Un mecanismo para conocer la importancia de la Biomímesis es la participación de la arquitectura en el desarrollo de Emprendimientos de Alto Impacto (EAI). Desde esta perspectiva, el objetivo de la investigación fue identificar como la bioinspiración al materializarse en el Biodiseño forma parte de la transformación integral y sistémica urbana en la región metropolitana de Bilbao, en Bizkaia (País Vasco, España) y en Bogotá Distrito Capital (D.C), Colombia. Para el estudio se hace uso de los principios de la Biomímesis (la naturaleza como medida, mentora y modelo), la manera como se expresa en los Biodiseños arquitectónicos de vanguardia y cómo se refleja en la economía circular. Los resultados obtenidos demuestran que inspirarse del lado positivo de la naturaleza trasciende en el contexto real, genera desarrollo inclusivo y auspicia una innovación que impacta en el bien común, así como la protección del medio ambiente con la puesta en marcha de una economía y sociedad del conocimiento.

\section{Introducción}

El proceso de urbanización del territorio en el mundo va acompañado de forma directa e indirecta con la dinámica del crecimiento poblacional mundial, el cual se proyecta que para el año 2050 alcanzará los nueve mil ochocientos millones de personas y seguirá creciendo en una proporción de ochenta y tres millones cada año (UN, 2017; Martín, 2017). Este crecimiento exponencial de la especie humana hace que se genere, cada vez más, una carga de presión de uso como de manejo, e impacta sobre el desarrollo de los componentes de los ecosistemas. Los componentes sean bióticos y abióticos son los que soportan la economía circular de la naturaleza, la economía humana y la coevolución de vida misma en la biosfera.

En este sentido, el propósito de este artículo es hacer una aproximación de la forma como se está implementado en el desarrollo urbano de las capitales Bilbao y Bogotá D.C., a partir de Emprendimientos de Alto Impacto basado en la biomimética y que buscan ordenamiento espacial sostenible, cultura del conocimiento y transformación urbana. Para ello, inicialmente se hace una descripción general de la Biomímesis, para posteriormente abordar lo relacionado con la economía circular y por último Emprendimiento de Alto Impacto (EAI), todo ello concerniente a los proyectos urbanos más destacados en relación a procesos de copiar a la naturaleza (transición biomimética) en esas dos urbes. 


\section{Metodología}

Para la investigación se utilizaron como unidades de análisis diseños bioinspirados de la naturaleza en las ciudades metropolitanas de Bilbao y Bogotá D.C. El criterio de elección se fundamentó en la conveniencia, ya que existían condiciones que facilitaban el acercamiento, a la recolección de información y a la observación; haciendo énfasis en la infraestructura urbana en transición biomimética en las dimensiones interrelacionadas de infraestructura, transporte, movilidad, educación, cultura, conservación y restauración ecosistémica; aspectos que dinamizan el proceso de transición biomimética en las dos metrópolis.

Para la recolección de información, se visitaron las instalaciones de tratamiento de los desechos sólidos y líquidos urbanos en Bilbao. Se hicieron recorridos de observación por el cinturón verde de Bilbao y de la zona forestal protectora de Bogotá D.C, siguiendo el sendero ecoturístico de la leyenda del "Venado Dorado". En la ciudad de Bogotá se contó con la visita a las áreas de transformación biomimética urbana, mediante un registro fotográfico y recogiendo información secundaria. Se reforzó la parte de conseguir la información con fuentes secundarias como estudios, marcos legales que han producido las instituciones que vienen desarrollando los proyectos de transformación urbana tanto en Bilbao como en Bogotá. Se contó con el apoyo de instituciones investigadores universidades de Colombia y del País Vasco que vienen trabajando en temas de Ecoinnovación y Biomímesis.

\section{Marco teórico conceptual}

\subsection{El paradigma biomimético}

La Biomímesis es una novedosa forma de hacer conocimiento, ciencia y tecnología (tecnociencia), que respeta la socio(bio)diversidad del mundo a partir del uso inteligente e inspirado en los componentes de la naturaleza. El cambio de paradigma desde la Biomímesis consiste en reconocer que la naturaleza tiene estructuras económicas, que deben ser copiadas, adaptadas a las empresas y a la economía de mercado capitalista. Se debe considerar a la economía humana un subsistema del sistema de la economía de la naturaleza en la biosfera terrestre. Los postulados centrales que rigen el paradigma de la Biomímesis, para lograr implementar una transformación real del sistema empresarial hegemónico, se deben acometer bajo las siguientes premisas: 1). Concebir a la naturaleza como su modelo; 2). Determinar a la naturaleza como medida; 3). La naturaleza como mentora. Actualmente la humanidad solo aprovecha el $10 \%$ de la tecnología de la naturaleza trasferida a la tecnología moderna humana, la cual aún es muy limitada. Se inicia una era basada, no en lo que podemos extraer del mundo natural, sino en lo que éste puede enseñarnos (Benyus, 2012,13).

\subsection{Economía circular de materiales y la Biomimesis.}

Al reconocer que la naturaleza ostenta unas estructuras económicas, las cuales existen desde hace 4.000 millones de años, la economía humana debe aprender de ellas e imitarlas, ya que la base de la economía de la naturaleza se relaciona con los procesos de circulación de materiales y el uso eficiente de la radiación solar; primado el proceso de reciclaje, de todos los productos de desecho (nutrientes) (Mitxelena y Gomez, 2017). Se fundamenta en un "sistema de circuito cerrado de ciclo de materiales movidos por el sol. En semejante "economía", la durabilidad se maximiza y los recursos terrestres en "teoría" pueden durar tanto como el sol siga irradiando la energía para mover los ciclos materiales cerrados" (Daly, 1989,30). Muchos materiales usados en las cadenas productivas en las empresas están rompiendo el ciclo natural, por vía de eutrofización, contaminación, toxicidad y la escasez de minerales como son las tierras raras; en lo que se puede considerar un impacto causado por el ser humano en una escala de tiempo corto. Fenómeno que se ve aún más agravado cuando se utilizan materiales no biodegradables, disipativos, ecotóxicos y se sintetizan compuestos de creciente complejidad, lo cual limita y hace más difícil lo relacionado con el reciclado y la reutilización (Bermejo, 2001,151, Bernal, 2015). 


\subsection{Emprendimiento de Alto Impacto (EAI).}

El Emprendimiento de Alto Impacto (EAI) es la forma integral en que una persona emprendedora (proyecto) consolida ideas de negocio. Se caracteriza por la manera sistémica como trasforma positivamente el desarrollo del entorno, dejando de lado el modelo empresarial tradicional en el que las prioridades son el tamaño y la rentabilidad, gestionando el interés monetario pero incorporando en sus objetivos beneficios a la comunidad con un rol social. Esta transformación se logra para cambiar las consecuencias que dejan el desdeño del emprendimiento capitalista y da la oportunidad de reconfigurar nuevos "modelos", que pasen de la autodestrucción a la sostenibilidad y de la desigualdad a la inclusión social. Así las cosas, el EAl, surge como respuesta para generar cambios disruptivos en la manera de hacer negocios e invita a las ciencias como la sociología, antropología y la biomimésis a incorporar de manera sistémica los resultados que están dejando los estudios científicos en desarrollo de productos responsables con el medio ambiente; que impacten en una economía circular y realmente se integren al mercado con conceptos de modelo de negocio. Esta integración contribuye a liberar el conflicto que ejerce la industria tradicional, con las nuevas economías, y proyecta la articulación de modelos de emprendimiento que promueven el uso sostenible de los recursos naturales enfocados en el bien común.

\section{Resultados y discusión.}

Los resultados de esta investigación se presentan con base en dos ciudades, región metropolitana de Bilbao y Bogotá D.C., a partir del desarrollo en los últimos treinta años de una serie de proyectos urbanos y de planificación del territorio, los cuales han incidido para lograr una transformación del paisaje urbano y rural adjunto. Para cada caso se hace una descripción general de las obras de renovación urbana, de restauración y de interconectividad ecosistémica del paisaje circundante en relación con la movilidad y el trasporte urbano, el manejo de los desechos urbanos, la educación, la cultura, el descanso, el entretenimiento y la inclusión social. Todo ello teniendo como perspectiva los procesos de emprendimiento que apoyan la transición biomimética del orden urbano, base para lograr una economía circular de materiales y del conocimiento.

\subsection{La ciudad de Bilbao.}

El área metropolitana de la ciudad de Bilbao se encuentra conformada por treinta municipios, donde viven más de un millón de personas. Hace apenas 30 años, Bilbao y el entorno del río Nervión que comunica esta ciudad con el mar Cantábrico (la Ría de Bilbao), era un área ocupada por la industria pesada. La villa de Bilbao era una ciudad gris y sucia a la que se iba a trabajar pero en la que se evitaba vivir. Con la crisis económica de los años 80, el Ayuntamiento de Bilbao diseñó un Plan General de Ordenación Urbana en el que se señalaban las áreas de futuro desarrollo, desplazando la industria hacia el puerto exterior, en la desembocadura del río Nervión; la figura 1 (izquierda), corresponde al área urbana de la ciudad de Bilbao. La industria se fue desplazando hacia el puerto exterior, en la desembocadura del río Nervión. La recuperación de los márgenes de la Ría para quienes habitan Bilbao y las poblaciones ribereñas han supuesto una transformación de la fisonomía de estas poblaciones y ha convertido a Bilbao en una ciudad sumamente atractiva tanto para habitar como para visitar; en la figura 1 (derecha) se puede apreciar la ciudad de Bilbao y su ria, destacándose el Museo Guggenheim, sede universidad del País Vasco, Universidad de Deusto, el edificio Iberdrola, el Palacio Euskalduna y el Estadio de San Mames.
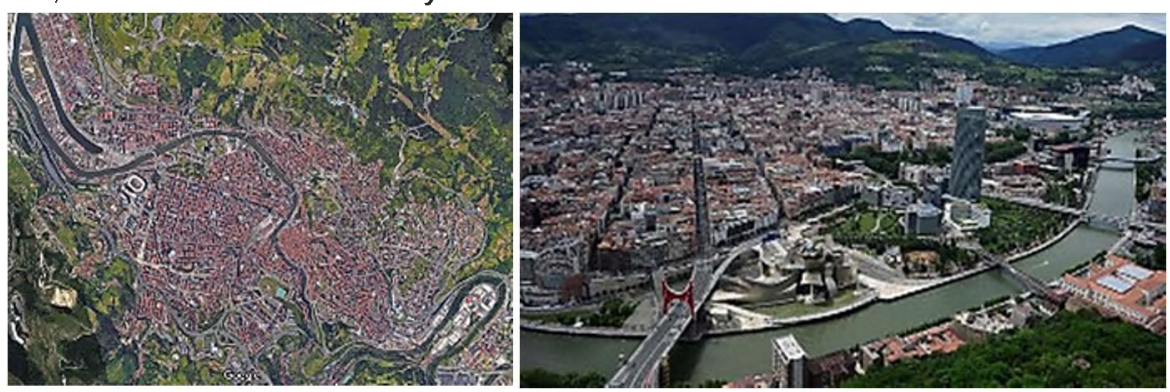

Fuente:https://www.google.com/maps/@43.2770678,-2.9513891,947 2.9513891,9479m/data=!3m1!1e3; Reuters - ABC

Fig. 1: La ciudad de Bilbao y el área de influencia de la ria. 


\subsubsection{Bilbao Ría 2000}

En el centro de todos estos cambios urbanos que ha sido objeto la ciudad de Bilbao está el Proyecto emblemático denominado "Bilbao Ría 2000", en el cual participan al 50\% las administraciones de la Comunidad Autónoma de País Vasco y de España. El proyecto "Bilbao Ría 2000" se hizo cargo del desarrollo de otras áreas a lo largo de la Ría, así como de ciertas infraestructuras; en la figura (2) se puede aprecia el tranvía y el proyecto de edifico más alto de la villa de Bilbao. Las principales áreas de actuación de la Sociedad son Abandoibarra, Ametzola, Bilbao La Vieja, Barakaldo y la zona de Basurto-San Mamés-Olabeaga. El proyecto actúa y ejecuta una serie de actuaciones del orden urbanismo, transporte, medio ambiente e inclusión social, con el propósito de regenerar y restaurar, sobre todo, aquellos espacios industriales no utilizados.

Las áreas de desarrollo de "Bilbao Ría 2000" que han dinamizado la ciudad se localizan en Abandoibarra, ocupada antes por astilleros y tinglados portuarios, siendo ahora un centro turístico de la ciudad que acoge, entre otros equipamientos de referencia internacional, al Museo Guggenheim Bilbao y al Palacio Euskalduna, el nuevo centro de congresos y de la música de la ciudad. La transformación no solo ha consistido en la renovación urbanística de estas localidades, también ha consistido en el paso de la actividad industrial a la de servicios, de unas ciudades degradadas y sucias a unas limpias y seguras. Esta transformación no se ha detenido, al continuar su dinámica con el compromiso de las administraciones públicas y privadas.

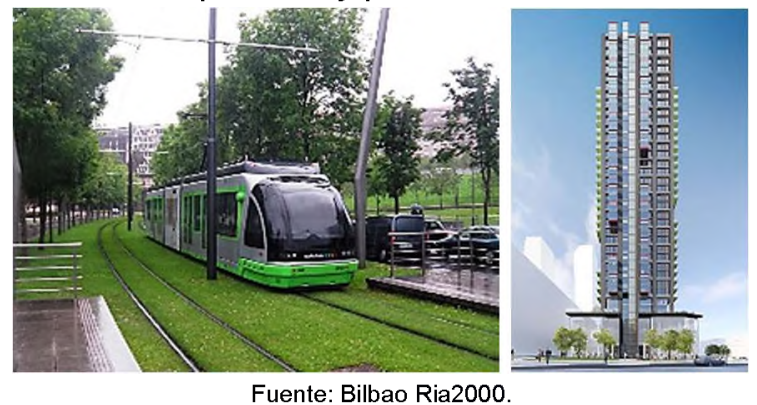

Fig 2. El Tranvía (izquierda) y La torre de Urbanización Garellano (derecha).

\subsubsection{Museo Guggenheim}

El Museo Guggenheim (figura 3), se puede considerar como una de las joyas de la arquitectura moderna de la ciudad Bilbao, es el icono de transformación económica, social y cultural de dicha ciudad, que la posiciona a nivel internacional. Es obra del arquitecto Frank Gehry y fue inaugurado en 1997. El edificio es majestuoso, imponente por donde se le aprecie, su apariencia desde la ría se relaciona desde la perspectiva de formar de un barco rindiendo homenaje a la ciudad portuaria en la que se inscribe. Visto desde arriba, sin embargo, el edificio posee la forma de una flor. Sus paneles brillantes de titanio se asemejan a las escamas de un pez, recordándonos las influencias de formas orgánicas biomiméticas. Es el atractor turístico nacional e internacional de la villa de Bilbao por excelencia.
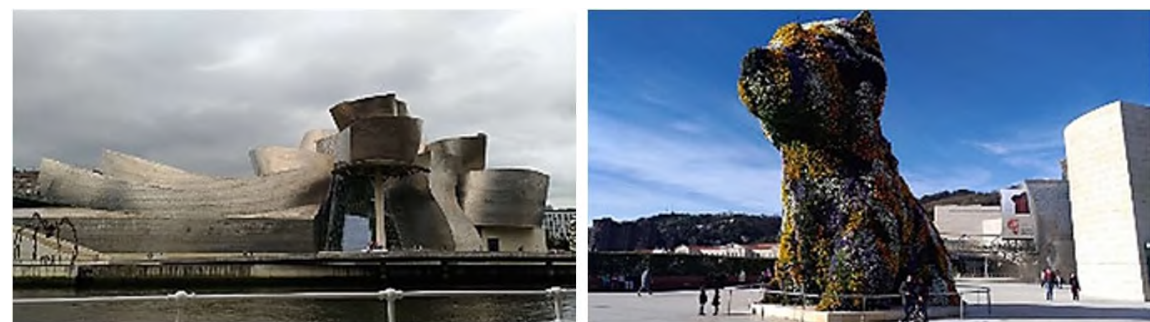

Fuente: Bernal Zamudio.

Fig 3. El museo Guggenheim visto desde la Ría (izq.) y entrada principal con la escultura ajardinada Puppy delante (der).

\subsubsection{Isla de Zorrotzaure: "Isla Creativa".}

El proceso de regeneración urbanística de la "Isla de Zorrotzaure" se desarrolla actualmente en 80 hectáreas, como se puede apreciar en la figura 4 (izq). Implica crear un barrio nuevo bien conectado con el resto de la ciudad de Bilbao y esta generando un nuevo paisaje urbano donde se plasma una 
arquitectura regenerativa. Esta transformación urbanística destaca por la combinación armónica entre lo moderno y lo antiguo, donde protege el patrimonio arquitectónico industrial y las nuevas perspectivas de la arquitectura moderna de vanguardia hecha por la arquitecta anglo-iraquí Zaha Hadid; como se puede apreciar en a figura 4 (der.). En la denominada "Isla Creativa", al día de hoy están instaladas 30 empresas y asociaciones culturales. En la isla de Zorrotzaure se construirá un Parque Tecnológico Urbano, un tranvía, un distrito financiero en la margen Sur, un polideportivo, un carril central para las bicicletas longitudinal y contará con amplias zonas verdes y parques recreaciones donde se construirán 5.500 viviendas de protección oficial.
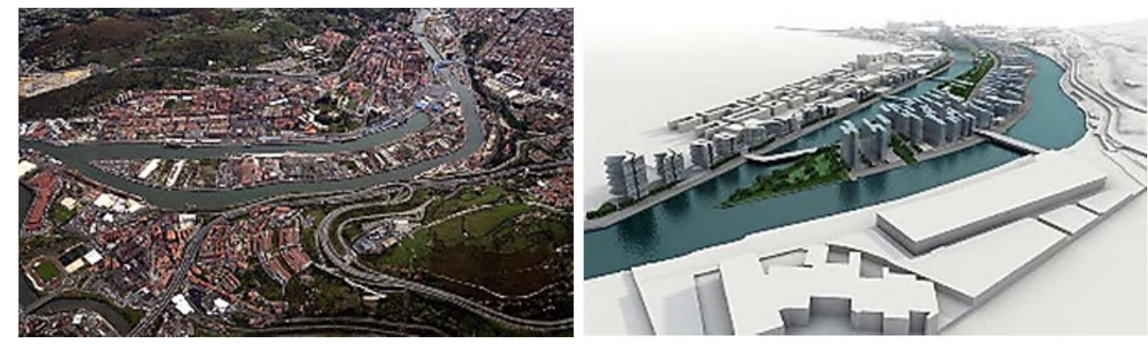

Fuente:https://bilbaoenconstruccion.com/2007/10/26/zorrozaurre-una-nueva-ciudad-dentro-de-una-isla/ Fig. 4. La isla de Zorrotzaure (izquierda) y Maqueta del proyecto urbano de "Isla creativa" (derecha).

\subsubsection{Ecoparque}

El Ecoparque se localiza en el monte de Arraiz, inicialmente llamada "Cantera Primitiva". La superficie a edificar es de $23.000 \mathrm{~m}^{2}$, incluyendo la Planta de Tratamiento Mecánico Biológico situada en Arraiz (Bilbao) la Planta de Compostaje (Konpostegia), la Planta de Valorización Energética (Zabalgarbi) y el depósito de Artigas, como se puede apreciar en la figura (5). Estas instalaciones conforman el "ecoparque" dedicado a la gestión de residuos sólidos y lixiviados que se producen, fundamentalmente, en la parte urbana del Gran Bilbao metropolitano.

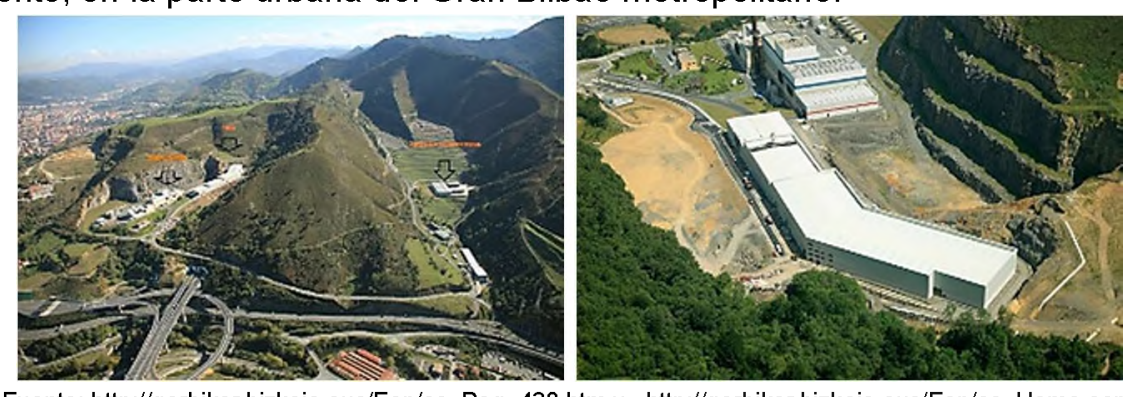

Fuente: http://garbiker.bizkaia.eus/Esp/ca_Pag_438.htm y http://garbiker.bizkaia.eus/Esp/ca_Home.asp

Fig. 5. Vista aérea de las instalaciones del Ecoparque de Monte de Arraiz.

El diseño de la planta está planificado para transportar 180.000 toneladas al año de la ciudad de Bilbao, con un proceso de clasificación mecánica y tratamiento biológico de la parte orgánica.

\subsubsection{Cinturón y/o anillo verde del Bilbao Metropolitano}

El "Cinturón Verde de Bilbao" tiene una década desde su implementación, con una extensión que va desde los $40 \mathrm{~km}$ hasta los 71.25 kilómetros por las conexiones viales. El objetivo inicial es ampliar la posible oferta de ocio en una ciudad y ampliar los espacios naturales para el ocio y la recreación. El recorrido permite disfrutar del paisaje rural y urbano, ya que atraviesa los parques forestales de Artxanda, Monte Avril, Arnotegi, Pagasarri y Arraiz, formando un sistema de caminos, senderos y calles, con sus respectivas señalizaciones; en la figura (6) se puede apreciar el cinturón verde y el plano de las rutas de los senderos. Se trata de espacios que conservan una serie de ecosistemas de gran valor: los ecosistemas costeros como el biotopo del humedal costero del Barbadún, las montañas que albergan pequeñas áreas de vegetación natural con una mayor extensión de cubierta forestal de plantaciones comerciales forestales (Onaindia, et al,. 2015). 

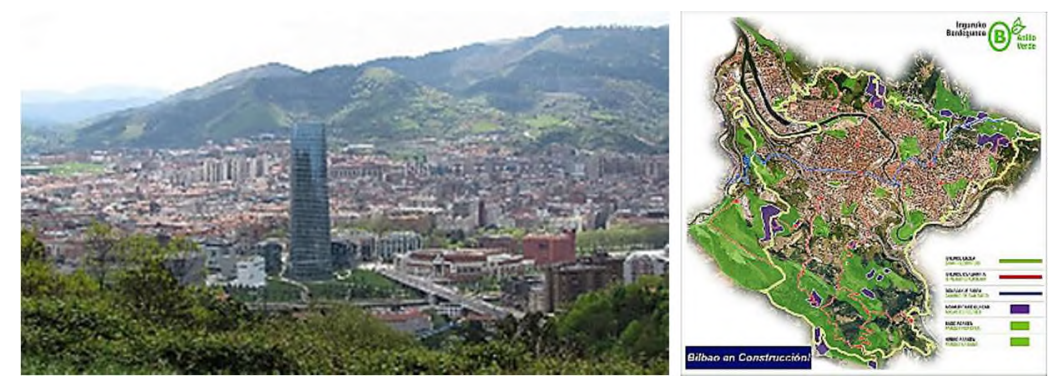

Fuente: https://desarmandobilbao.files.wordpress.com/2014/10/anillo-verde-bilbao.jpg Fig.6. Cinturón verde de Bilbao (izquierda) y el mapa de las rutas (derecha).

\subsection{La ciudad de Bogotá D.C.}

La ciudad de Bogotá D.C se localiza en un valle interandino, como se puede apreciar en la figura 7 (izq.). En la actualidad tiene una población de 8 millones de personas que la habitan permanentemente y de unas 10 millones de personas consideradas población flotante. Dicho espacio socio-biogeográfico es muy cosmopolita, cohabitando diferentes grupos culturales, ya que conecta a todo el Estado nación, y es el centro neurológico de la toma de decisiones en las áreas económica, política, cultural de la nacionalidad colombiana y de interacción dinámica con el resto del mundo; en la figura 7 (der.), se aprecia la parte central de la ciudad ded Bogotá. El proceso de transformación urbanística de esta mega ciudad es relevante $y$, aunque se puede describir de manera general a partir de las obras públicas relacionadas con la movilidad, la protección medio ambiental, la difusión cultural, la educativa, económica y la técnico-científica; en este caso se le da prioridad a la dimensión medio ambiental.

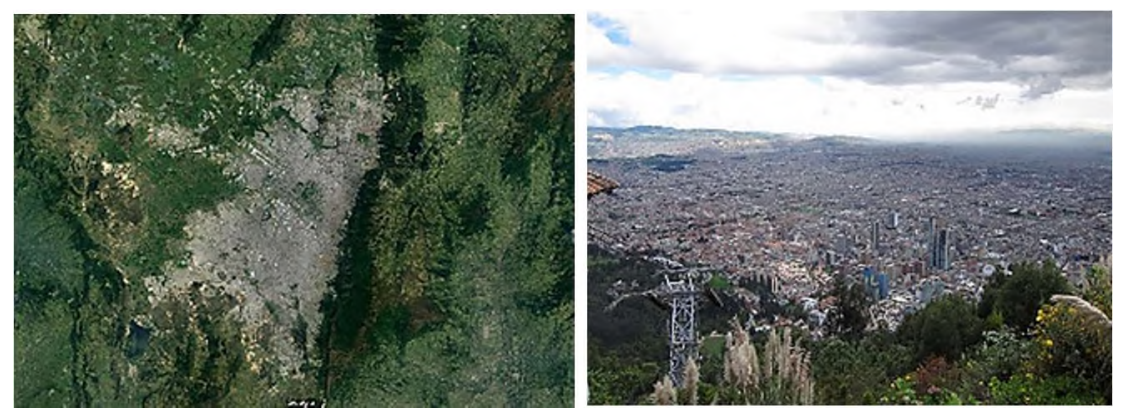

Fuente: google earth y Becerra, 2016

Fig. 7. Las ciudad de Bogotá D.C (izq) y Área central de Bogotá (der).

\subsubsection{Parque Metropolitano Simón Bolívar}

Uno de los primeros procesos de desarrollo urbanístico biomimético es el Parque Simón Bolívar, el cual se convirtió en el pulmón verde de la ciudad de Bogotá. Se trata de un espacio construido donde convergieron necesidades humanas relacionadas con el esparcimiento, el tiempo de ocio, sumideros de $\mathrm{CO}_{2}$ y un refugio para la vida y flora nativa. En la figura (8) destaca la Biblioteca Virgilio Barco, la infraestructura deportiva y de recreación del Parque Salitre, el Jardín Botánico y el Parque Simón Bolívar.
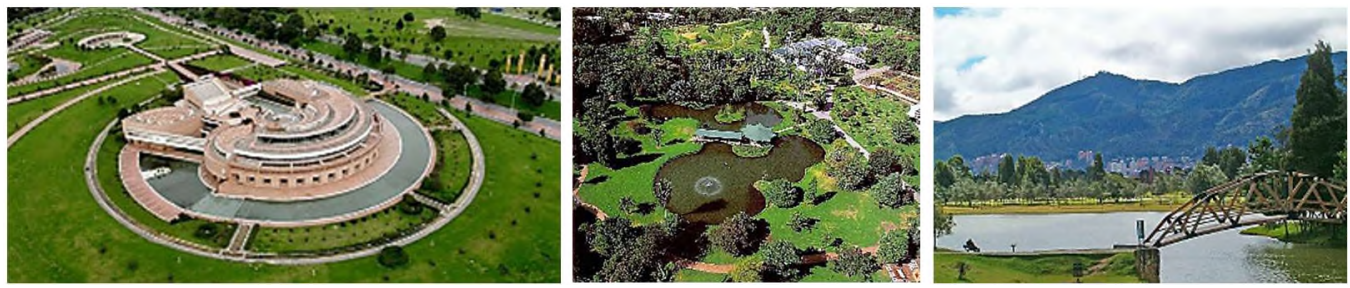

Fig. 8. Biblioteca Virgilio Barco (izq), Jardín Botánico (centro) y Parque Metropolitano Simón Bolívar (der).

\subsubsection{Restauración y reforestación de las avenidas emblemáticas}

Es importante resaltar el proceso de arborización de sus avenidas emblemáticas como son la Carrera Séptima, la Carrera 30, la Avenida 68, Calle 26, la Avenida Boyacá, Avenida de las Américas, 
Autopista del Sur, Autopista Norte, Avenida Primero de Mayo, Avenida Caracas, entre otras. Se convierten dichas avenidas en corredores biológicos longitudinales y trasversales de la metrópoli; son barreras vivas contra la contaminación acústica, de aire, suelo, agua y mejoran ostensiblemente el paisaje urbano; son ecosistemas construidos para refugio de la fauna y flora nativa, ya que son espacios de alimentación, reproducción y de estación de paso de las aves migratorias. En la figura (9) se pueden apreciar tres de las grandes avenidas en proceso de restauración y arborización de Bogotá (Reforestación Avenida el Dorado; Avenida Alo; Avenida el Ferrocarril).

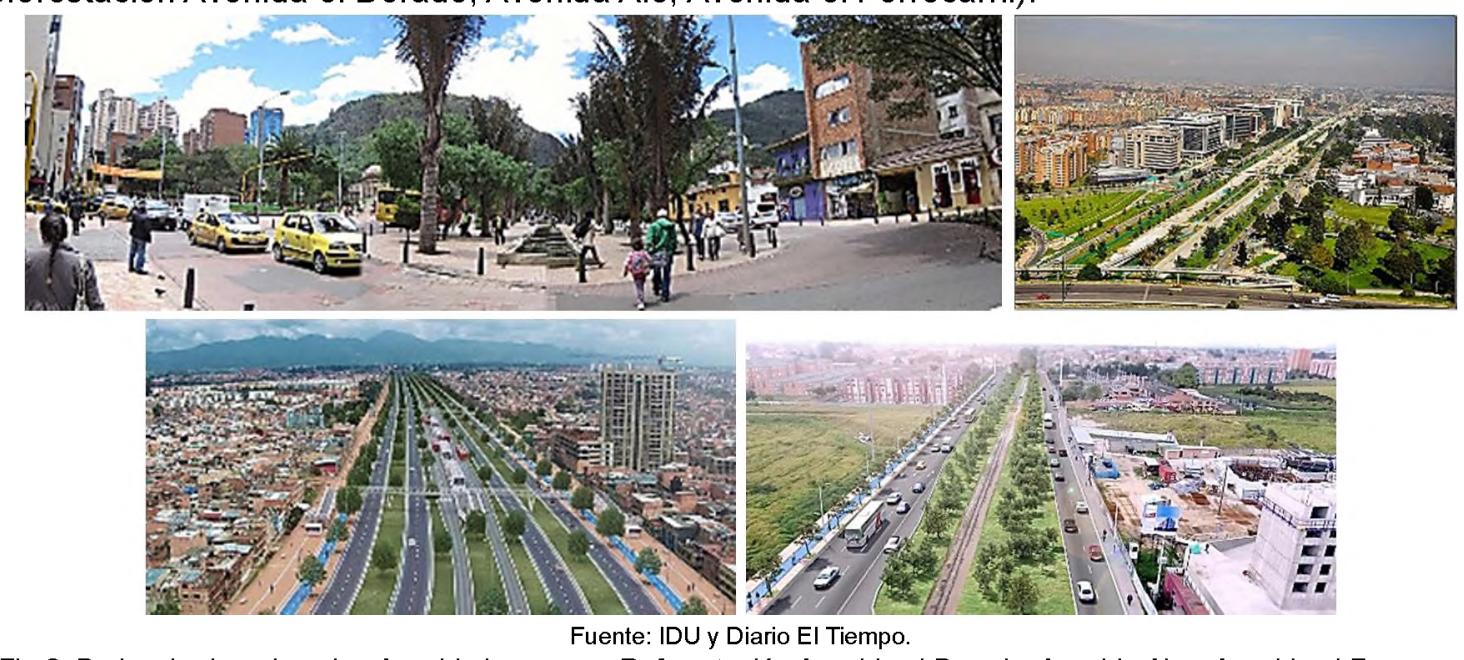

Fig 9. De izquierda a derecha: Avenida las aguas, Reforestación Avenida el Dorado; Avenida Alo y Avenida el Ferrocarril.

\subsubsection{Remodelación del Parque de los Martires}

El parque se creó en 1850 con el fin de conmemorar la terminación de la guerra de los 1000 días, y se encuentra en todo el centro de la ciudad. A partir de este espacio urbano, se ha desarrollado un proceso de trasformación urbana del centro de la ciudad muy integral y sistémico, ya que se conecta con el norte el sur, este y oeste de la ciudad de Bogotá D.C. Esta remodelación permite dirigir acciones encaminadas a impulsar la economía relacionada con el turismo, los servicios, la innovación y las finanzas. Se fundamenta en la construcción de un centro financiero, con zonas verdes arborizadas. Se integra con el sistema de "transmilenio" a partir de la estación de la Avenida Jiménez. Al cruzar la Avenida Caracas, se encuentra el Parque Tercer Milenio que se construyó en el año 2000 en los terrenos del antiguo El Cartucho. En la figura (10) se puede apreciar la nueva plaza del Parque de los Mártires.

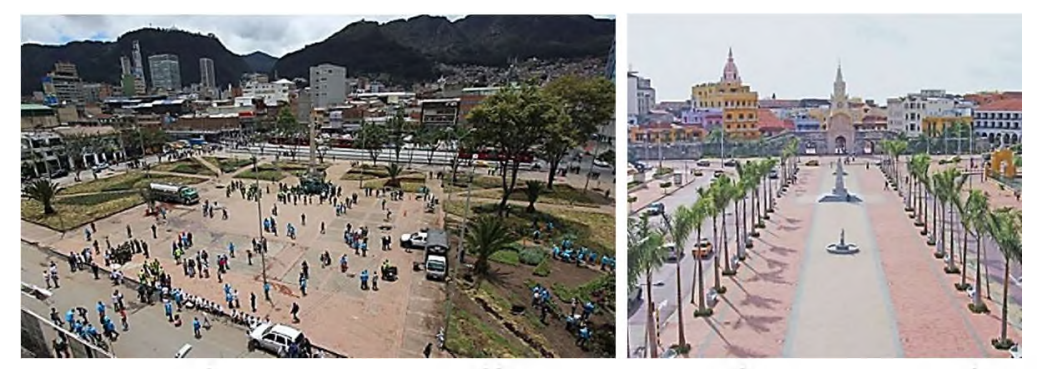

Fuente: Secretaria de Cultura, Recreación y Deporte, Alcaldia Mayor de Bogotá D.C

Fig. 10. Conexión en la Plaza de los Mártires al Transmilenio (izquierda) y remodelación del parque de los Mártires (der).

\subsubsection{Ciclo vías y ciclo rutas}

Una de las formas de movilidad e interconectividad de la ciudad son las ciclovías y ciclorutas que unen los barrios con las poblaciones de la sabana y de otros departamentos limítrofes. Esta vías son una forma de hacer la ciudad inclusiva y verde. En la actualidad se tienen 120 kilómetros de ciclorutas nuevas y comodidades peatonales para el transporte diario. En la figura (11), se pueden apreciar las ciclovía y cicloruta por la Avenida del Dorado. El diseño de las vías siguen la dirección de la infraestructura vial principal imperante y de nuevas rutas que permiten recorrer los humedales y senderos que se han construido a propósito en los parques nacionales naturales de Chingaza y 
Zumapaz, con el fin de que se conozca y proteja la flora y la fauna de la sabana, los páramos andinos.

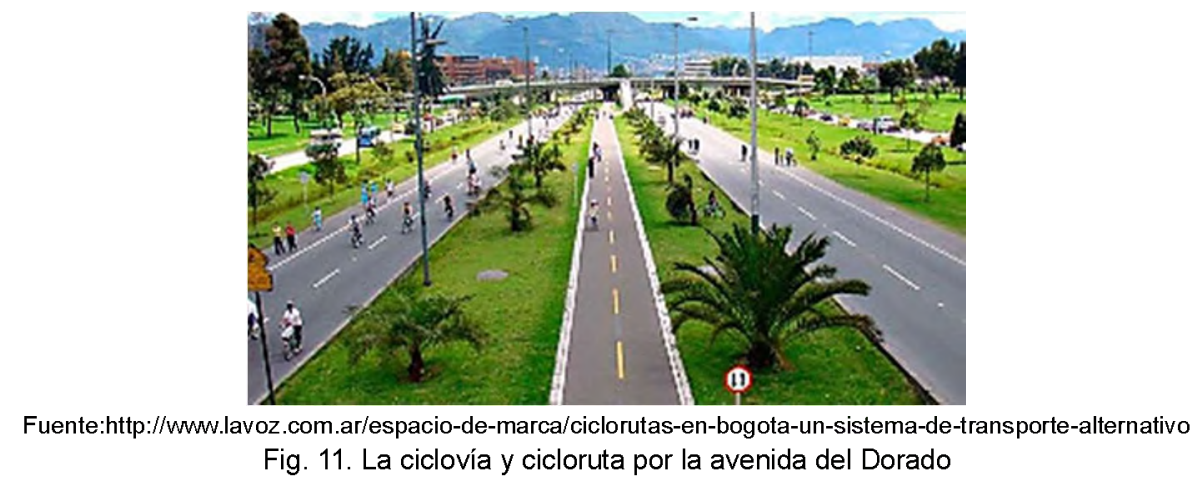

Las ciclovías y ciclorutas recorren la ciudad de norte a sur, de este a oeste, y a partir de estos espacios que mantienen unas coberturas vegetales en forma de arbolados y jardines, se convierten en zonas verdes que hacen la función de corredores y conectores biológicos, los cuales mitigan la contaminación urbana. A nivel de esparcimiento y tiempo de ocio de la ciudadanía bogotana, cumplen la función de educar a la ciudadanía sobre la importancia de las zonas verdes con el fin de practicar deportes, promoviendo la buena salud.

\subsubsection{Trasmilenio}

"Transmilenio" es un sistema de transporte masivo que se implementó en Bogotá en el año 2000. El sistema comenzó a funcionar con autobuses articulados de color rojo que constan de dos cuerpos unidos por un fuelle. Para su movilización, tienen carriles exclusivos a través de la ciudad con rutas por la calle 80, la troncal de la Caracas, la Autopista Norte, la Avenida Suba, la NQS (Avenida Carrera 30), la calle 26, la Autopista Sur, el Eje Ambiental, la carrera 10, la carrera 7, la Avenida de las Américas y la Autopista Sur. En el año 2004 la ciudad recibió el premio de la Unesco denominado "Ciudades por la Paz", por reducir la inseguridad, promover espacios urbanos, impulsar la vida cultural y por el sistema de transporte publico de "TransMilenio". En la figura (12) se puede apreciar el transmilenio y la cicloruta paralela.

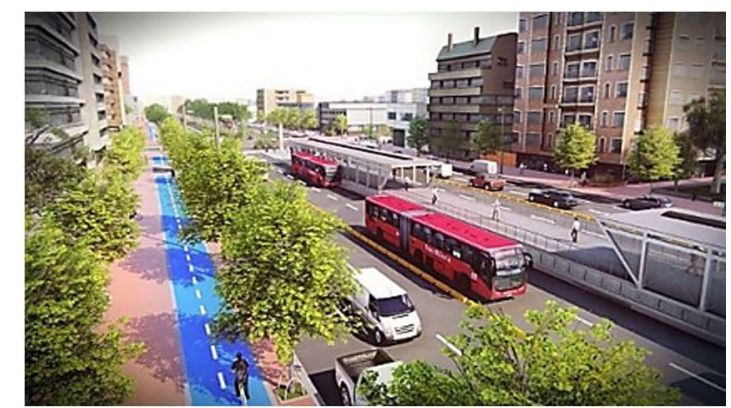

Fig. 12. Sistema de trasporte público Transmilenio y una ciclovía paralela

\subsubsection{Barrio de la Candelaria universitaria y cultural}

La ciudad de Bogotá se fundó en siglo XVI (6 de agosto de 1538) y hoy ostenta una zona colonial llamada "La Candelaria", con una extensión de 184 has. Espacio arquitectónico que aún mantiene las grandes casas con interiores ajardinados y fuentes hídricas, similares a las construcciones mediterráneas de Andalucía y Extremadura en España; en la figura (13), se puede apreciar una de las calles de la zona colonial de la Candelaria. En este espacio se encuentran universidades de gran prestigio, museos, iglesias, plazoletas, restaurantes, asociaciones culturales y parques. Destaca la Fuente de Quevedo. La Candelaria se está convirtiendo en un núcleo universitario, administrativo publico distrital, nacional y cultural. Esta zona se ha visto favorecida en su nuevo ordenamiento espacial por la peatonalización y jardines de la carrera Séptima, la rehabilitación de las plazoletas del Centro Cultural Gabriel García Márquez, Gregorio Vásquez de Arce y Ceballos. 

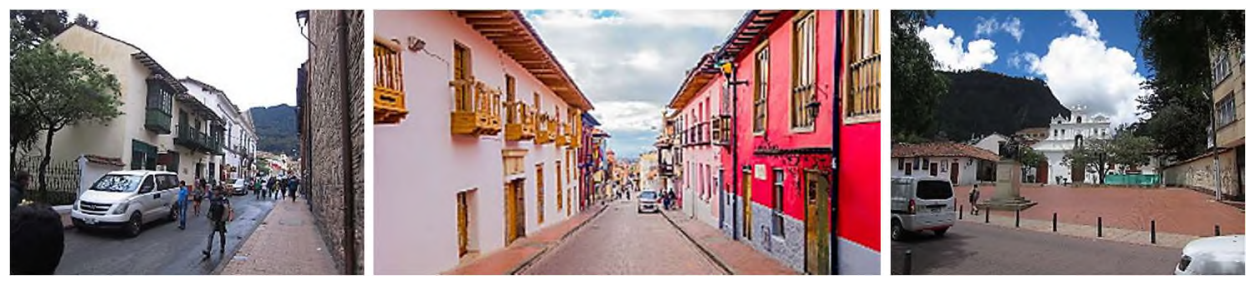

Fuente: Becerra, 2016

Fig. 13. Calles del barrio la Candelaria

La implementación del sistema de transporte masivo Transmilenio mejoró la conectividad con el resto de la ciudad por medio de la construcción de las estaciones: Las Aguas, Museo del Oro, (avenida Jiménez), Bicentenario y San Victorino (carrera Décima). Se pretende, además, hacer una acción de integración y mejoramiento con otros barrios vecinos como son Teusaquillo, Santa Fe, Mártires, Centro Administrativo, La Catedral, La Concordia y Egipto (Manrique, 2013).

\subsubsection{Cinturón verde y humedales}

Los cerros de Monserrate y Guadalupe son los guardianes de la capital y cumplen la función de ser el corredor biológico de las estribaciones cordilleranas de los Andes con la planicie de la sabana de Bogotá y están dentro de la categoría de "Reserva Forestal Protectora"; en la figura (14) se puede apreciar el paisaje y los monumentos arquitectónicos religiosos de Monserrate y Guadalupe con sus respectivas zonas forestales. Ambientalmente, son muy importantes, ya que en su espacio descargan las masas de nubes que provienen del Magdalena medio y sus estribaciones permiten drenar sus aguas de lluvia a los riachuelos, quebradas, lagunas y humedales que van posteriormente al casco urbano de la ciudad.

Los humedales de Bogotá son 33 y ocupan 667 hectáreas. Resulta importante destacar que han sido declarados 11 humedales como zona RAMSAR en el año 2018 (SDA, 2018). Son los últimos remanentes de ecosistemas donde se refugia la fauna nativa con especies endémicas. Se pretende que sea una vía de conexión con los corredores biológicos de la sabana con el rio Bogotá con los bosques de la reserva forestal.

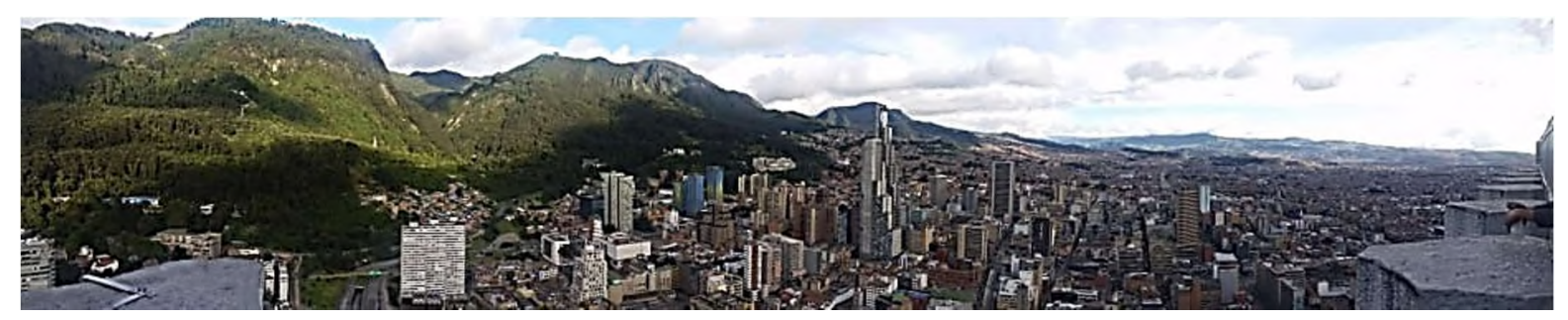

Fuente: Bernal, 2018

Fig. 14. Los cerros de Monserrate y Guadalupe, vista panorámica de Bogota centro oriental.

\subsubsection{Nuevas edificaciones verdes}

Entre las nuevas edificaciones que se están construyendo en la capital que cumplen con los criterios de sostenibilidad encontramos el edificio verde de Santalaia localizado en la calle 76 con $4 \mathrm{~A}$ en el barrio Chapinero. En su fachada exterior como en los interiores cuenta con 115.000 plantas; en la figura (15) se puede apreciar la cubierta vegetal del edificio Santalaia. Es de resaltar la constante innovación en términos de implementar bioarquitectura a partir de coberturas vegetales que generan un nuevo paisaje urbano y cumplen con la función de ser sumideros de $\mathrm{CO} 2$. Este estilo de arquitectura verde permite el proceso de limpiar las emisiones de carbono que producen aproximadamente unos 745 autos, lo cual es una estrategia para mitigar y adaptarse al cambio climático, además de cumplir la función de ser refugio de fauna silvestre y flora andina. 


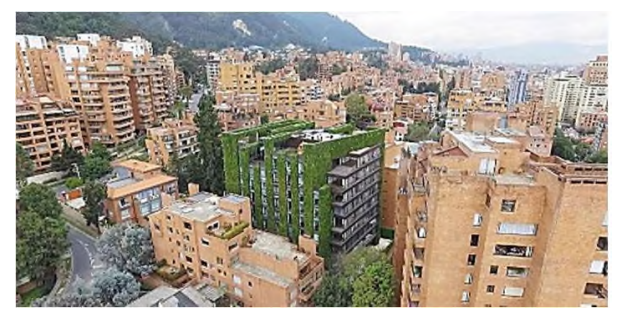

Fuente: http://www.eltiempo.com/bogota/el-edificio-verde-que-oxigena-al-barrio-rosales-87482

Fig. 15. Edificio verde de Santalaia con su respectiva cubierta vegetal.

Este edifico contiene un jardín vertical con azoteas ajardinadas de más de 3.117 metros cuadrados. La filosofía consiste en "donde el concreto (hormigón) sustituyó a la naturaleza, ahora la naturaleza debe sustituir al concreto", imitando el paisaje que nos ofrecen las coberturas vegetales. La función biomimética del edificio es la de descontaminar y enriquecer el sector con oxígeno para aproximadamente 3.000 habitantes. El impacto visual y del pasaje es formidable. Se pretende construir además"La Ciudad Conecta" y el proyecto urbanístico de la fábrica de Bavaria Ciudad Kennedy, proyectos arquitectónicos verdes, como se puede apreciar en las figura (16).
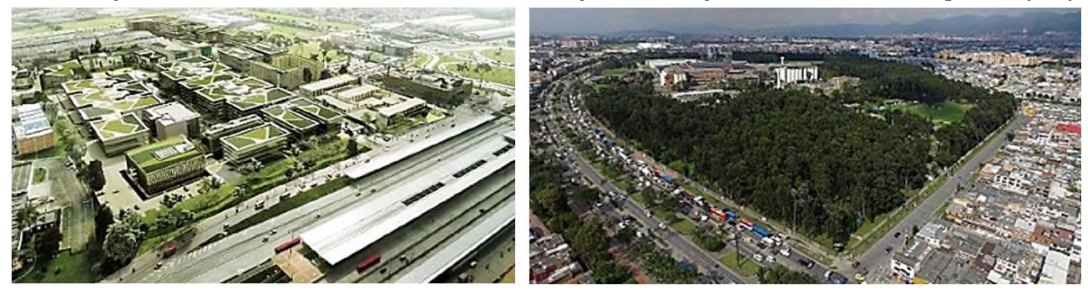

Fuente:http://www.jllproperty.com.co/es-co/bogot\%C3\%A1/salitre-eldorado/oficinas-en-arriendo/edificio-connecta-oficinas-en-arriendo-enbogot\%C3\%A1/339423_y https://www.civico.com/bogota/noticias/10-obras-que-le-cambiaran-la-cara-a-la-calle-26

Fig 16. El proyecto CONNECTA (izquierda) y Predio actual para el proyecto urbanístico de Fábrica de Bavaria (derecha)

\section{Conclusión}

El proceso de urbanización concentra una de las mayores tasas de disipación de materiales y de energía producidas de forma antrópica. Dicho impacto pasa por el no cierre de los ciclos de vida de los materiales, como del uso ineficiente de las energías renovables y la restricción al acceso de la información de vanguardia, generando procesos de inconmensurabilidad. Los procesos de aplicación directa e indirecta de la biomimética a la trasformación urbana de las ciudades de Bilbao y Bogotá. D.C, según su contexto, se puede catalogar de estratégico. Con el impacto positivo de la aplicación de la biomimética a la arquitectura en el desarrollo del Emprendimiento de Alto Impacto (EAI), se intenta imitar tanto los procesos como las funciones de los componentes de la naturaleza. Esta circunstancia se aprecia en el impacto sobre la recuperación, restauración de los ecosistemas naturales relacionados con los bosques (en forma de cinturones verdes, humedales, riachuelos y quebradas que se transforman en corredores biológicos urbanos y rurales), los cuales han sido intervenidos para que de nuevo cumplan su función de ser resilientes. Al implementarse la Biomimética, se va construyendo una economía circular, una sociedad del conocimiento inclusiva.

Lo que se pudo constatar de forma negativa para la ciudad de Bogotá D.C y Bilbao, es el no cierre del ciclo de los materiales relacionado con los desechos sólidos y líquidos urbanos, que aun no son vistos como nutrientes, por lo que no se vislumbra un cierre del ciclo de materiales en el corto plazo y aun es más grave para los barrios de Bogotá, ya que no se hace una selección de los materiales en los hogares. Para el caso de Bilbao, aun las aguas de la ria siguen con problemas de contaminación por los sedimentos depositados en su cause de origen industrial histórico.

El desarrollo de la infraestructura del tren de alta velocidad en Bilbao y del metro de Bogotá traerán un cambio muy significativo al ordenamiento urbano en cada una de las ciudades y su área de influencia metropolitana. Para el caso de Bogotá se complementa con los siguientes proyectos urbanos como son la "Ciudad CAN", la Ciudad Empresarial Sarmiento Angulo, el Centro Empresarial Elemento, el Central Point, la ampliación del Aeropuerto El Dorado, el Museo Nacional de Memoria, los rascacielos de las Torres Atrio, el Parque Bicentenario, la Facultad de Artes y Diseño de la Universidad Jorge 
Tadeo Lozano, el Parque Fontanar y la remodelación logística del parque el Tunal.

\section{Referencias Bibliográficas.}

Benyus, J. (2012). Biomímesis. Cómo la ciencia innova inspirándose en la naturaleza. TUSQUETS EDITORES. Barcelona, España.

Bermejo, R. (2001). Desarrollo sostenible y humano. P. Ibarra, \& K. Unceta, Ensayos sobre el desarrollo humano. Barcelona: Icaria.

Bernal, Z, H. (2015). Aproximación a un enfoque biomimético para la creación de sociedades amazónicas del bienestar sustentable. Universidad del País Vasco.

Daly, H. (1989). Economía, ecología y ética. Ensayos hacia una economía en estado estacionario. FCE, México.

EL TIEMPO. (2017). El edificio verde que oxigena el barrio Rosales de Bogotá. http://www.eltiempo.com/bogota/el-edificio-verde-que-oxigena-al-barrio-rosales-87482. Consultado vía web el día 22/07/2018.

Manrique, G, A.S. (2013). Gentrificación de La Candelaria: reconfiguraciones de lugar de residencia y consumo de grupos de altos ingresos. Cuadernos de Geografía: Revista Colombiana de Geografía. Volumen 22, Número 2, p. 211-234, 2013.

Martín, B. (2017). En datos: así seremos 2050. El País. https://elpais.com/elpais/2017/11/06/ciencia/1509960076_506979.html. Consultado vía web el 20/07/2018.

Mitxelena, A, Gomez, E, (2017). From ecological architecture to biomimicry. Journal of Sustainable Architecture and Civil Engineering, Número 20, p. 68-74, Kaunas University of Technology, Kaunas, 2017.

Onaindia, M, Madariaga, I, Palacios, Igone, Arana X, (2017). Naturaleza y Bienestar en Bizkaia: la evaluación de los servicios de los ecosistemas; investigación aplicada a la gestión. Universidad del País Vasco.

La Voz. (2015). Ciclo rutas en Bogota, un sistema de trasporte alternativo. http://www.lavoz.com.ar/espacio-de-marca/ciclorutas-en-bogota-un-sistema-de-transportealternativo.Consultado vía web el 29/07/2018.

United Nation (UN). (2017). World population prospects 2017. https://esa.un.org/unpd/wpp/. Consultado vía web el 20/07/2018.

Secretaría Distrital de Ambiente (SDA). 2018. Los humedales de Bogotá entran en la lista de importancia internacional RAMSAR. Consultado vía Web el día 03/08/2018. 